\title{
White dwarf pollution by hydrated planetary remnants: Hydrogen and Metals in WD J204713.76-125908.9
}

\author{
Matthew J. Hoskin, ${ }^{1,2 \star}$ Odette Toloza, ${ }^{1}$ Boris T. Gänsicke,,${ }^{1,2}$ Roberto Raddi, ${ }^{3,4}$ \\ Detlev Koester, ${ }^{5}$ Anna F. Pala, ${ }^{6,1}$ Christopher J. Manser, ${ }^{1}$ Jay Farihi, ${ }^{7}$ \\ Maria Teresa Belmonte, ${ }^{8}$ Mark Hollands, ${ }^{1}$ Nicola Gentile Fusillo, ${ }^{6,1}$ Andrew Swan ${ }^{7}$ \\ ${ }^{1}$ Department of Physics, University of Warwick, Coventry, CV4 7AL, UK \\ ${ }^{2}$ Centre for Exoplanets and Habitability, University of Warwick, Coventry, CV4 7AL, UK \\ ${ }^{3}$ Dr. Remeis-Sternwarte 85 ECAP, Friedrich-Alexander Universität Erlangen-Nürnberg, Sternwartstr. 7,96049 Bamberg, Germany \\ ${ }^{4}$ Departament de Fisica, Universitat Politécnica de Catalunya, c/Esteve Terrades 5, E-08860 Castelldefels, Spain \\ ${ }^{5}$ Institut für Theoretische Physik und Astrophysik, Christian-Albrechts-Universität, 24118 Kiel, Germany \\ ${ }^{6}$ European Southern Observatory, Karl Schwarzschild Straße 2, Garching, 85748, Germany \\ ${ }^{7}$ University College London, London, WC1E 6BT, UK \\ ${ }^{8}$ Blackett Laboratory, Physics Department, Imperial College London, London SW7 2AZ, UK
}

Accepted XXX. Received YYY; in original form ZZZ

\begin{abstract}
WD J204713.76-125908.9 is a new addition to the small class of white dwarfs with helium-dominated photospheres that exhibit strong Balmer absorption lines and atmospheric metal pollution. The exceptional abundances of hydrogen observed in these stars may be the result of accretion of water-rich rocky bodies. We obtained farultraviolet and optical spectroscopy of WD J204713.76-125908.9 using the Cosmic Origin Spectrograph on-board the Hubble Space Telescope and X-shooter on the Very Large Telescope, and identify photospheric absorption lines of nine metals: $\mathrm{C}, \mathrm{O}, \mathrm{Mg}$, $\mathrm{Si}, \mathrm{P}, \mathrm{S}, \mathrm{Ca}, \mathrm{Fe}$ and Ni. The abundance ratios are consistent with the steady state accretion of exo-planetesimal debris rich in the volatile elements carbon and oxygen, and the transitional element sulphur, by factors of seventeen, two, and four respectively compared to bulk Earth. The parent body has a composition akin to Solar System carbonaceous chondrites, and the inferred minimum mass, $1.6 \times 10^{20} \mathrm{~g}$, is comparable to an asteroid $23 \mathrm{~km}$ in radius. We model the composition of the disrupted parent body, finding from our simulations a median water mass fraction of eight per cent.
\end{abstract}

Key words: stars: abundances - white dwarfs - planetary systems - stars: individual: WD J204713.76-125908.94

\section{INTRODUCTION}

White dwarfs are remnants of main sequence stars less massive than $\simeq 8 \mathrm{M}_{\odot}$, which have shed their envelope during their post-main sequence evolution to leave a cooling degenerate star. Their high surface gravities $(\log g \simeq 8)$ cause chemical stratification, so their atmospheres are dominated by either hydrogen or helium. Studies of increasing sensitivity have shown that the majority of helium atmosphere white dwarfs below $\simeq 20,000 \mathrm{~K}$ contain detectable atmospheric hydrogen, but insufficiently abundant to form a superficial radiative hydrogen layer (Fontaine \& Wesemael 1987; Eisenstein et al. 2006; Voss et al. 2007; Bergeron et al. 2011; Koester \& Kepler 2015; Rolland et al. 2018). Spectra of these stars can

^ E-mail: M.Hoskin@warwick.ac.uk show absorption lines from both elements, and their relative abundance, $\log [\mathrm{H} / \mathrm{He}]^{1}$, can be determined from model atmosphere analysis. In most cases where both elements are present, helium is the dominant element, with $\log [\mathrm{H} / \mathrm{He}]$ ranging from $10^{-1}$ to $10^{-7}$ or lower.

Spectral evolution of white dwarfs between hydrogenand helium-dominated atmospheres has been studied extensively; however, the origin of hydrogen remains contentious (e.g. Koester 1976; Fontaine \& Wesemael 1987; MacDonald \& Vennes 1991; Beauchamp et al. 1996; Voss et al. 2007; Tremblay \& Bergeron 2008; Koester \& Kepler 2015; Gentile Fusillo et al. 2017; Rolland et al. 2018; Cunningham et al. 2020). Throughout the literature, trace hydrogen in helium atmospheres has been ascribed to: (i) convective processes 
dispersing hydrogen that survived giant branch evolution (Herwig et al. 1999; Althaus et al. 2010; Koester \& Kepler 2015; Rolland et al. 2018, 2020); (ii) episodic or continuous accretion from the interstellar medium (ISM: Koester 1976; MacDonald \& Vennes 1991; Voss et al. 2007); (iii) water-rich planetesimal accretion (Farihi et al. 2013; Veras et al. 2014b; Raddi et al. 2015; Gentile Fusillo et al. 2017; Xu et al. 2017).

Rolland et al. (2018, 2020) showed that many measured $\log [\mathrm{H} / \mathrm{He}]$ values in their sample are incompatible with evolution involving either a constant mass of (primordial) hydrogen, or a constant rate of hydrogen accretion, since the white dwarf formed. However, along with the presence of trace hydrogen, trace metals are commonly seen in the atmospheres of helium (and hydrogen) atmospheres white dwarfs (e.g. Koester \& Kepler 2015; Gentile Fusillo et al. 2017). Unlike hydrogen, which persists in the upper atmosphere, metals sink below the photosphere on timescales between $10^{-2}$ $10^{7} \mathrm{yr}$ (Koester 2009). The consensus is that these metals originate in the star's planetary system; from rocky bodies which have been captured and accreted.

Planetesimals perturbed onto orbits that cross the Roche radius of the white dwarf will undergo tidal disruption and form a debris disc, before being accreted (Jura 2003, 2008; Metzger et al. 2012; Veras et al. 2014a, 2015). Debris discs have been detected at infrared wavelengths as an excess of flux in tens of systems (e.g. Graham et al. 1990; Kilic \& Redfield 2007; von Hippel et al. 2007; Debes et al. 2011; Girven et al. 2012; Brinkworth et al. 2012; Rocchetto et al. 2015; Farihi 2016), and through emission lines from gaseous metals in a few cases (e.g. Gänsicke et al. 2006, 2007, 2008; Farihi et al. 2012a; Melis et al. 2012).

Once accreted, metals give rise to narrow absorption lines which are detectable in spectroscopy (Jura \& Young 2014; Hollands et al. 2017). The bulk composition of the disrupted parent body can be obtained by modelling the atmospheric abundances to reproduce these spectroscopic lines, and the calculation of gravitational diffusion timescales for each element. For atmospheres with effective temperature $T_{\text {eff }} \gtrsim 25000 \mathrm{~K}$, radiative levitation can sustain metals at detectable abundances and so must also be considered (Chayer et al. 1995). The variety in deduced compositions is comparable to our own Solar System (e.g. Koester et al. 2005; Zuckerman et al. 2007; Jura 2008; Melis et al. 2011; Gänsicke et al. 2012; Xu et al. 2013), and recent works have shown that this diversity can be explored quantitatively in terms of planet formation theory (Harrison et al. 2018; Swan et al. 2019b) and Solar System chemistry (Doyle et al. 2019).

The possibility of detecting the accretion of water-rich planetary material was first raised by Jura et al. (2009). More recent and extensive modelling - including factors such as formation, stellar progenitor mass and treatment of both heat and mass transport (Malamud \& Perets 2016, $2017 \mathrm{a}, \mathrm{b})$ - has demonstrated that a large fraction of the total water content ${ }^{2}$ of a rocky body can survive. Farihi et al. (2013) first reported a white dwarf with atmospheric metal abundances consistent with excess oxygen - indicative of water rich material - and evidence of similar accretion episodes has since been found (Raddi et al. 2015; Farihi et al. 2016).

\footnotetext{
2 Including both water ice and hydrated minerals - throughout
} this paper, we will simply refer to "water" to refer to both.
Furthermore, eight of the 17 polluted white dwarfs examined by Harrison et al. (2018) are best fitted by a parent body containing significant amounts of water, with five of these at $3 \sigma$ statistical significance. This demonstrates that some water sequestered in planetesimals survives into the later stages of stellar evolution, in line with the prediction of Jura \& Xu (2010). Hydrogen atoms from dissociated water will remain in the white dwarf's atmosphere indefinitely, and is an observable indicator that water-rich material may have been accreted.

A statistical analysis of helium atmosphere white dwarfs found a highly significant correlation between photospheric metal pollution and the presence of trace hydrogen, interpreted as evidence that hydrogen is accreted alongside planetary debris - most likely as water Gentile Fusillo et al. (2017). This is a compelling indicator that accretion of rocky planetary bodies can contribute to photospheric and spectral evolution of white dwarfs. Further, it shows that in some cases hydrogen should be considered as an external pollutant alongside the metals often seen in white dwarf atmospheres.

There are seven known white dwarfs with a photosphere composed mainly of helium, but containing sufficient hydrogen that the Balmer lines dominate their spectral classification, and with existing observations that allow identification of atmospheric pollution: GD 16 (Koester et al. 2005); GD 362; PG 1225-079 (both Xu et al. 2013); SDSS J124231.06+522626.7 (Raddi et al. 2015); WD 1536+520 (Farihi et al. 2016); WD 1425+540 (Xu et al. 2017); GD 17 (Gentile Fusillo et al. 2017). Multiple metals are identified in each of these stars, from which the compositions of accreted planetary material are measured. This small family provides a valuable opportunity to probe the evolution of white dwarf atmospheres and investigate connections to planetesimal accretion.

In this paper we report on a new member of this family: WD J204713.76-125908.9 (adopting the new white dwarf naming convention outlined by Gentile Fusillo et al. 2019, hereafter referred to as WD J2047-1259). This star was identified to have a hydrogen atmosphere (O'Donoghue et al. 2013, as EC 20444-1310), until Raddi et al. (2017, as APASS J2047-1259) showed that Balmer and helium absorption lines are both present in optical spectra, and used $\log [\mathrm{H} / \mathrm{He}]=-1$ to analyse the spectrum. Neither O'Donoghue et al. (2013) nor Raddi et al. (2017) had observations with the required spectral coverage and resolution to probe for the presence of metals. In Section 2, we present follow-up spectroscopy taken with the Hubble Space Telescope $(H S T)$ and the Very Large Telescope (VLT), and in Section 3, we fit the atmospheric parameters of WD J20471259. In Section 4, we discuss the composition and nature of the accreted material, and in Section 5 consider the infrared data and possibility of a dusty disc. Section 6 comprises further discussion of the spectral evolution of helium atmosphere white dwarfs and of WD J2047-1259, and in Section 7 , we summarise our findings and their implications.

\section{OBSERVATIONAL DATA}

\subsection{Spectroscopy}

Owing to the extraordinary hydrogen abundance measured by Raddi et al. (2017), WD J2047-1259 was included in the 
target list for our HST snapshot program (ID: 15073, Cycle $25)$ to search for photospheric pollution. A short exposure (2000 s) far-ultraviolet (FUV) spectrum was obtained with the Cosmic Origins Spectrograph (COS) on 2017 November 04 using the G130M grating, centred at $1291 \AA$, with the instrument in Lifetime Position 4. This set-up provides wavelength coverage of $1130-1431 \AA$ (with a small gap at $1274-1287 \AA$ ) and resolving power $R \simeq 16000$.

To mitigate the fixed pattern noise that is affecting the COS FUV detector, we divided the exposure time equally between two FP-POS positions ( 1 and 4; the limited snapshot visit duration did not allow us to use all four positions). This spectrum reached a signal-to-noise of 15 , dominated by the broad Ly $\alpha$ absorption feature. A visual inspection identified strong absorption lines of silicon, carbon, oxygen, iron sulphur, phosphorus, and nickel.

To increase sensitivity - particularly in the Ly $\alpha$ wings and wavelength coverage, we carried out observations of a five-orbit mid-cycle GO program on 2018 November 18 (ID: 15474, Cycle 26). Two orbits used the G130M grating centred at $1291 \AA$, in lifetime positions 3 and 4 ( 1 and 2 were unavailable). The remaining three orbits used a central wavelength of $1222 \AA$, and the exposure time was divided equally across all four lifetime positions. At this central wavelength the Ly $\alpha$ core and the airglow emission fall onto the detector segment gap $(1211-1222 \AA)$. The wavelength coverage of this mode is $1064-1368 \AA$, and the resolving power slightly lower at $R \simeq 15000$. HST COS spectra were co-added and rebinned into a single spectrum with resolution elements of $0.05 \AA$, achieving a signal-to-noise of 38 in the continuum. This spectrum is shown in the upper panel of Fig. 1. Time-tag data were visually inspected and show no variability.

On 2018 July 10, we obtained a deep optical spectrum using X-shooter (Vernet et al. 2011), an echelle medium resolution spectrograph mounted on the Cassegrain focus of UT2 at European Southern Observatory's Very Large Telescope (ESO VLT) in Cerro Paranal (Chile). We used slit widths of $1.0,0.9$, and $0.9 \operatorname{arcsec}$ in the blue (UVB, $3000-5600 \AA$, $\mathrm{R}=5400$ ), visual (VIS, $5500-10200 \AA, \mathrm{R}=8900$ ) and nearinfrared (NIR, $10200-24800 \AA, \mathrm{R}=5600$ ) arms and exposed for $4 \times 1220,4 \times 1250$, and $4 \times 1300 \mathrm{~s}$, giving median signal-tonoise ratios of 212,126 , and 53 , respectively. The NIR arm was read out using the non-destructive mode to mitigate the effect of overexposed sky lines. Data were reduced using the Reflex pipeline (Freudling et al. 2013), which includes the standard reduction steps of bias and dark current removal, order identification and tracing, flat-fielding, dispersion solution, correction for instrument response and atmospheric extinction, and merging of all orders. Flux calibration was achieved using observations of a standard star and the wavelength scales were corrected for the barycentric radial velocity. Finally, a telluric correction was performed using molecfit (Smette et al. 2015; Kausch et al. 2015). Absorption lines from calcium, magnesium, oxygen and iron, along with the broad Balmer and helium lines, are visible in the UVB and VIS arms, and are highlighted in Fig. 2.

\subsection{Photometry}

We have compiled the photometry of WD J2047-1259 in Table 1. Data were taken from the GALEX (Bianchi et al.
Table 1. Photometric data for WD J2047-1259.

\begin{tabular}{llc}
\hline Survey & Filter & Brightness [AB mag] \\
\hline GALEX & $F U V$ & $15.531 \pm 0.012$ \\
APASS & $N U V$ & $15.521 \pm 0.008$ \\
& $B$ & $15.91 \pm 0.08$ \\
& $g^{\prime}$ & $15.86 \pm 0.03$ \\
& $V$ & $15.89 \pm 0.06$ \\
Pan-STARRS & $r^{\prime}$ & $16.05 \pm 0.05$ \\
& $i^{\prime}$ & $>15.97$ \\
& $g$ & $15.901 \pm 0.003$ \\
& $i$ & $16.180 \pm 0.002$ \\
Gaia & $z$ & $16.466 \pm 0.003$ \\
& $y$ & $16.718 \pm 0.005$ \\
2MASS & $B P$ & $16.875 \pm 0.009$ \\
& $G$ & $15.965 \pm 0.009^{a}$ \\
VHS & $R P$ & $16.126 \pm 0.008^{a}$ \\
& $J$ & $17.35 \pm 0.010^{a}$ \\
& $H$ & $>16.40$ \\
Spitzer & $K_{S}$ & $>16.49$ \\
& $Y$ & $16.903 \pm 0.008$ \\
& $J$ & $17.318 \pm 0.013$ \\
& $K$ & $18.34 \pm 0.06$ \\
& $3.6 \mu \mathrm{m}$ & $19.33 \pm 0.05$ \\
& $4.5 \mu \mathrm{m}$ & $19.79 \pm 0.05$ \\
\hline
\end{tabular}

${ }^{a}$ Corrected using Maíz Apellániz \& Weiler (2018).

2011), Gaia (Gaia Collaboration et al. 2016), APASS (Henden et al. 2015), Pan-STARRS (Chambers \& Pan-STARRS Team 2016), 2MASS (Cutri et al. 2003) and VHS (Sutherland et al. 2015) online catalogues.

WD J2047-1259 was observed twice by the Spitzer Space Telescope (Werner et al. 2004) during the postcryogenic mission. Observations in each warm IRAC channel were taken separately: at $3.6 \mu \mathrm{m}$ on $2012 \mathrm{Jan} 06$, and at 4.5 $\mu \mathrm{m}$ on 2011 Jun 22 (both from program 80063, PI: Boyer). The field was observed with 18 dithered frames using $30 \mathrm{~s}$ exposures during each visit. The target is well exposed and, owing to the small dithering scale, is well covered in the image stack at 90 arcsec from the edge of the mosaic. The target lies 4.6 arcsec from an unrelated star with late Mdwarf colours based on Gaia and 2MASS, and 5.0 arcsec from a background galaxy based on its diffuse IRAC image.

Mosaics with 0.6 arcsec pixel ${ }^{-1}$ were created from the corrected basic calibrated data frames using the MOPEX package, following standard observatory recommendations. The flux was measured both using apertures and with the APEX module to perform point-spread function fitting simultaneously on the target and neighbouring sources. Aperture and array-location-dependent corrections were made, but pixel-phase and color corrections were ignored. The fluxes measured with an $r=4$ pixel ( 2.4 arcsec, 2 native pixel) radius aperture are likely contaminated by the neighbouring $\mathrm{M}$ star $(114 \pm 12$ and $66 \pm 7 \mu \mathrm{Jy}$ for the $3.6 \mu \mathrm{m}$ and $4.5 \mu \mathrm{m}$ band passes respectively), while values reported by APEX appear consistent with the model predictions for the stellar photosphere $(67 \pm 4$ and $44 \pm 3 \mu \mathrm{Jy})$. Photometric errors are the quadrature sum of the measurement error and a calibration uncertainty of 5 per cent. 


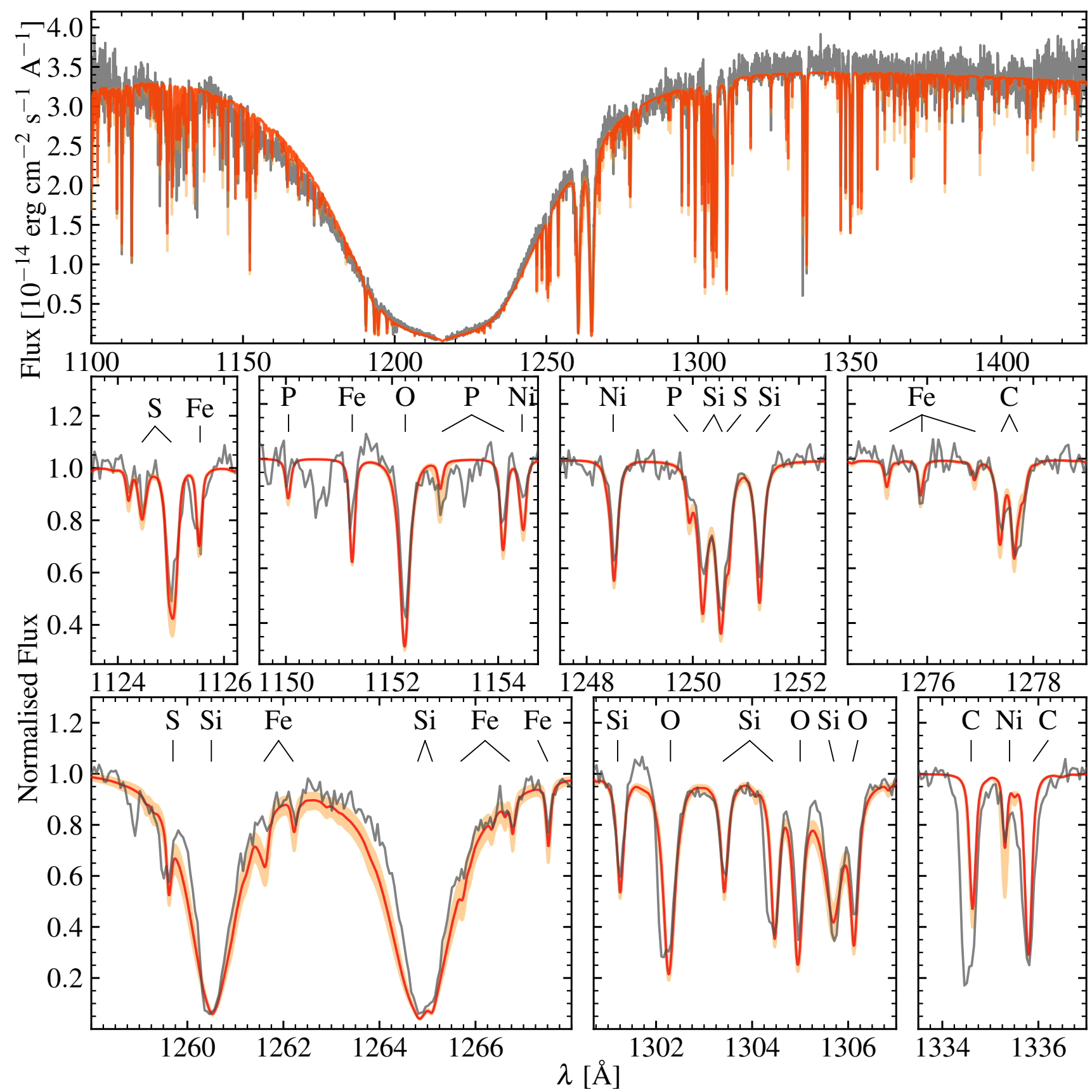

Figure 1. Atmospheric model (red, with $\pm 1 \sigma$ uncertainty in orange) and the rebinned COS spectrum (grey). The top panel shows the quality of the overall fit, which notably deviates from the level of the continuum below $1190 \AA$ (see main text). The middle and lower rows of panels show the strongest absorption lines of the atmospheric metals. The $1334.53 \AA$ C II line is clearly blended with a significant ISM absorption line.

\section{SPECTROSCOPIC FITTING}

We first undertook a rigorous fit to the atmospheric parameters, $T_{\text {eff }}, \log g$, and $\log [\mathrm{H} / \mathrm{He}]$, using a bespoke model grid computed with the code of Koester (2010). Once these parameters were finalised, their values were fixed during a visual fitting of the atmospheric metals.

\subsection{Atmospheric Parameters}

Our grid of synthetic spectra covers $10000 \leq T_{\text {eff }} \leq 20000 \mathrm{~K}$ in steps of $200 \mathrm{~K}, 7.0 \leq \log g \leq 9.0$ in steps of $0.1 \mathrm{dex}$, and $-5.0 \leq$ $\log [\mathrm{H} / \mathrm{He}] \leq 0.0$ in steps of $0.2 \mathrm{dex}$, and uses ML2 $/ \alpha=1.0$. These models do not include metals. We used the $\chi^{2}$ minimisation routine (scipy.optimize.fmin) from Jones et al. (2001) and tri-linearly interpolated synthetic spectra from our model grid. For each synthetic spectrum, we computed 


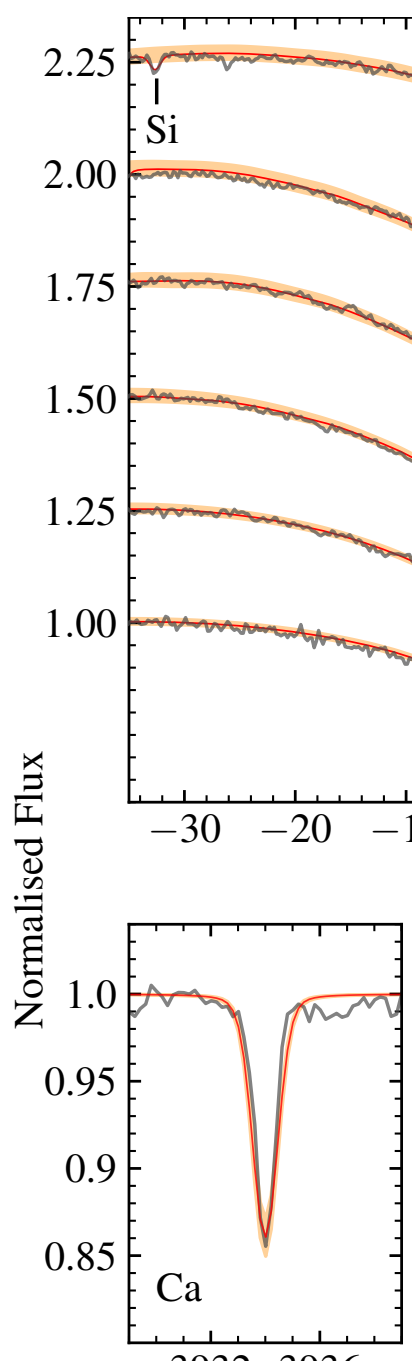

39323936

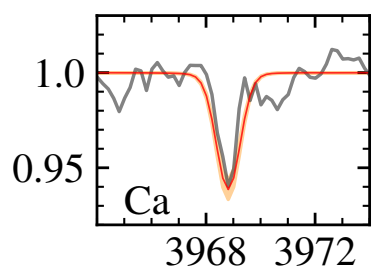

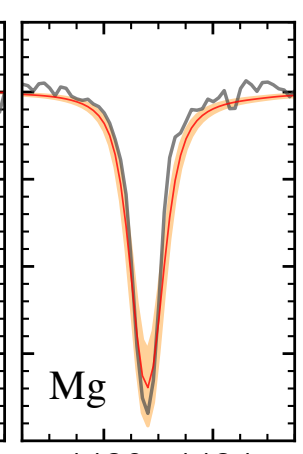

44804484
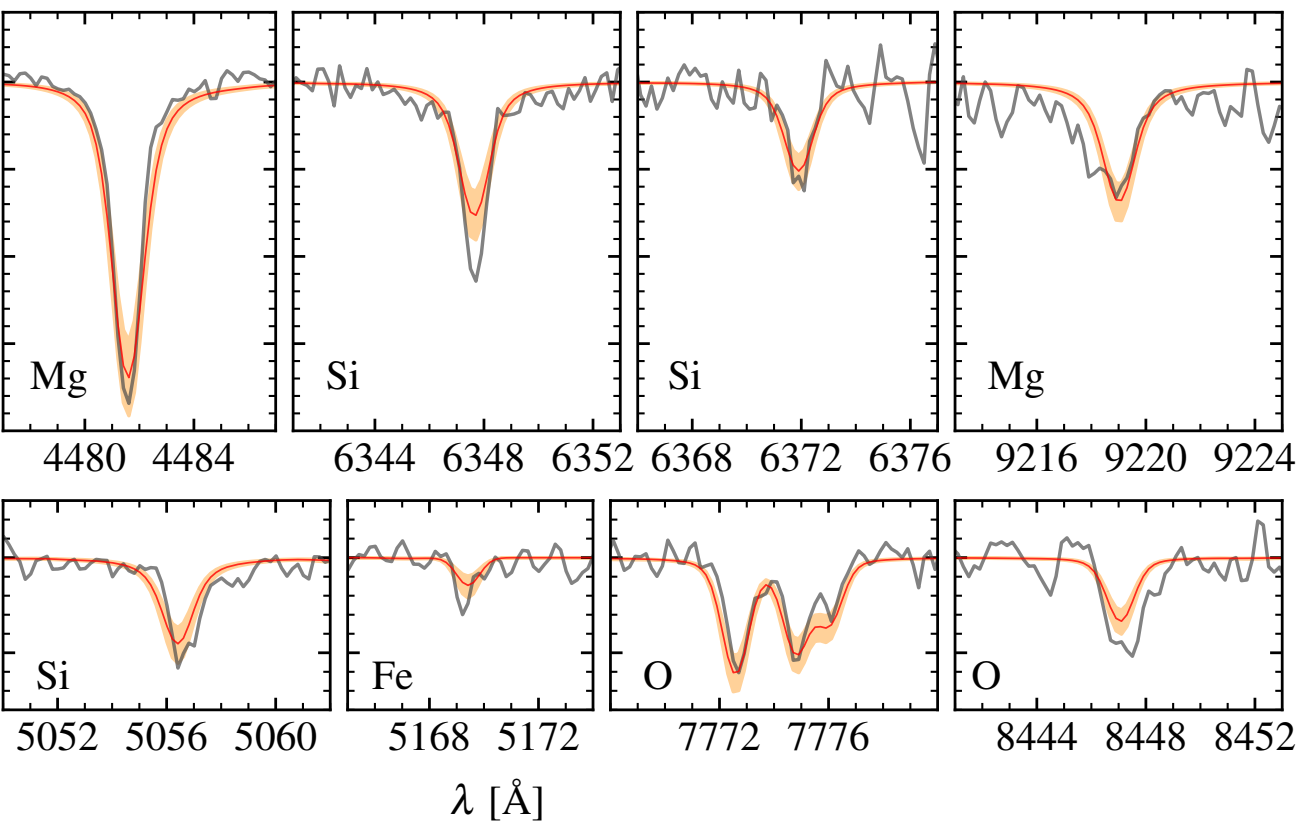

Figure 2. Atmospheric model (red, with $\pm 1 \sigma$ uncertainty in orange) and the normalised X-shooter spectrum (grey). The top panels show the normalised Balmer (left) and HeI (right) features, labelled by name or by central wavelength, where the shaded region shows the uncertainty on the normalised model. Absorption lines from metal ions are also labelled. The middle and lower rows of panels highlight several strong metal absorption lines. In these panels the shaded region is associated with the uncertainty in metal abundances.

the white dwarf radius, $R_{\mathrm{WD}}$, for the corresponding $T_{\mathrm{eff}}$ and $\log g$ using the mass-radius relation ${ }^{3}$ of Fontaine et al. (2001), and then applied a flux scaling factor of $S=R_{\mathrm{WD}}^{2} / d_{\varpi}{ }^{2}$, where $d_{\varpi}$ is the Gaia parallax distance.

WD J2047-1259 has a parallax distance of just under $100 \mathrm{pc}$ and so the effect of reddening is expected to be low. Local 3D dust maps yield an extinction of $E(B-V)=0.005 \pm$

\footnotetext{
${ }^{3}$ http://www.astro.umontreal.ca/ ${ }^{\text {bergeron/CoolingModels/ }}$
}

$0.017 \mathrm{mag}$ (Lallement et al. 2014; Capitanio et al. 2017; Lallement et al. 2018). We performed the fits described below using $E(B-V)=\{0.00,0.01,0.02,0.03\} \mathrm{mag}$ and reddened the synthetic spectra using the global mean extinction law of Fitzpatrick (1999). We found the photometric and spectroscopic fits were most consistent for $E(B-V)=0.01$, and we adopted this value for the final analysis.

We simultaneously fitted the COS spectrum (with metal absorption lines masked) and the normalised Balmer and 


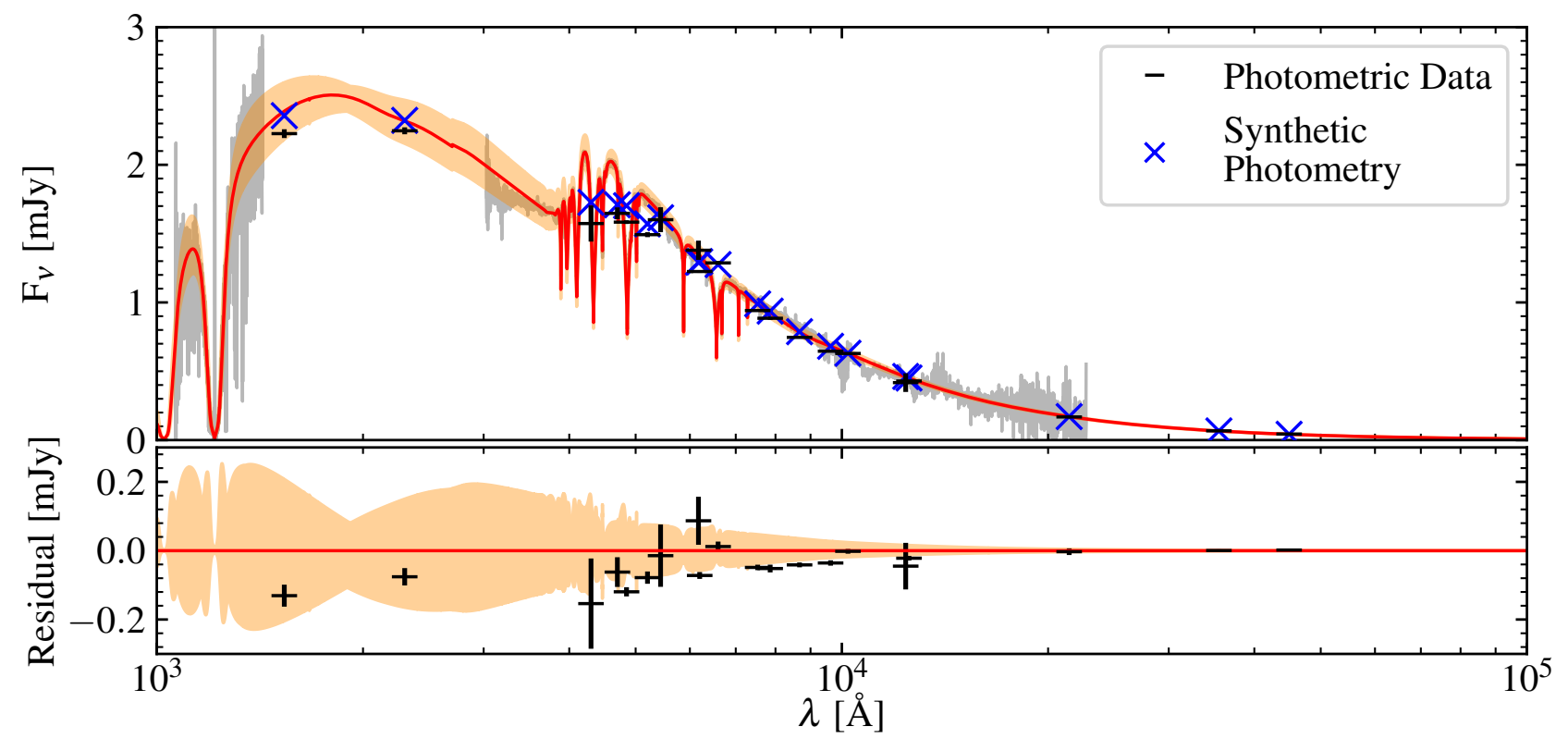

Figure 3. Spectral energy distribution of WD J2047-1259. From the model (red), which is the best-fit to the spectra (grey), synthetic photometry (blue crosses) are calculated and compared to survey photometry (black dashes). Most of the photometric data lie below the best-fitting model, but most are close to or within the $1 \sigma$ uncertainties in the spectroscopic fit.

helium line profiles in the X-shooter data ${ }^{4}$ to obtain a selfconsistent three-parameter $\left(T_{\text {eff }}, \log g, \log [\mathrm{H} / \mathrm{He}]\right)$ fit. We draw 1000 data sets $^{5}$ from a Monte Carlo simulation incorporating uncertainties from spectral extraction, and parallax for the un-normalised COS spectrum. The scatter in $T_{\text {eff }}, \log g, \log [\mathrm{H} / \mathrm{He}]$ were used to estimate a Gaussian error on each parameter. The best-fitting atmospheric parameters measured from the above procedure are $T_{\text {eff }}=17970_{-170}^{+140}$, $\log g=8.04 \pm 0.05$ and $\log [\mathrm{H} / \mathrm{He}]=-1.08 \pm 0.08$. The spectroscopic model (including metals, discussed in Section 3.2) and data are shown in Fig. 1 and Fig. 2. The Pearson correlation coefficient between $T_{\text {eff }}$ and $\log g$ is $\rho=0.99$, and between those and $\log [\mathrm{H} / \mathrm{He}], 0.573$ and 0.596 respectively. These atmospheric parameters are broadly consistent with the result of the pre-Gaia spectroscopic fit with $\log [\mathrm{H} / \mathrm{He}]=-1$ fixed (Raddi et al. 2017).

We fitted models to the photometry in Table 1 with the Gaia-based distance to check for consistency, but fix $\log [\mathrm{H} / \mathrm{He}]=-1.08$ as broad-band photometry is poorly suited to constraining the hydrogen abundance. We excluded Spitzer data in case of an infrared excess from a debris disc, which we discuss in Section 5. This recovers $T_{\text {eff }}=17920 \pm 60 \mathrm{~K}$ and $\log g=8.076 \pm 0.005$ with a Pearson correlation coefficient of $\rho=0.98$, which is consistent with our spectroscopic fit. The purely statistical errors on the photometric data do not represent the true uncertainties in ground based photometry. Similarly GALEX and Gaia

\footnotetext{
4 The near-infrared arm data are not used in our fitting procedure.

5 We varied the number of samples over three orders of magnitude ( $n=100$ to 10000 ), and found $n=1000$ gave results and distributions consistent with $n=10000$, so for computational expediency we used $n=1000$.
}

Table 2. Stellar properties of WD J2047-1259.

\begin{tabular}{ll}
\hline Parameter & Value \\
\hline$\varpi$ & $10.17 \pm 0.08 \mathrm{marcsec}$ \\
$d_{\varpi}$ & $98.3 \pm 0.8 \mathrm{pc}$ \\
$T_{\mathrm{eff}}$ & $17970_{-170}^{+140} \mathrm{~K}$ \\
$\log g$ & $8.04 \pm 0.05$ \\
$\log [\mathrm{H} / \mathrm{He}]$ & $-1.08 \pm 0.08$ \\
$\log q^{a}$ & -8.92 \\
$\mathrm{t}_{\mathrm{cool}}$ & $125 \mathrm{Myr}$ \\
$\mathrm{M}_{\mathrm{WD}}$ & $0.617 \mathrm{M}_{\odot}$ \\
$\mathrm{R}_{\mathrm{WD}}$ & $0.0131 \mathrm{R}_{\odot}$ \\
\hline
\end{tabular}

${ }^{a} \log q=\log \left(M_{\mathrm{cvz}} / M_{*}\right) ;$ mass fraction of the convection zone

photometry have been closely scrutinised, and significant corrections have been suggested for each (Maíz Apellániz \& Weiler 2018; Wall et al. 2019, respectively). A comparison of the photometric data with the synthetic photometry from the spectroscopic model is shown in Fig. 3. Table 2 lists properties of WD J2047-1259; the cooling age assumes a thin envelope (Fontaine et al. 2001).

We tested for discrepancies in $\log [\mathrm{H} / \mathrm{He}]$ determined from the ultraviolet and optical spectra by fitting the hydrogen abundance with each wavelength range individually, with $T_{\text {eff }}$ and $\log g$ fixed to the values in Table 2 , and at the $\pm 1 \sigma$ values. The best-fitting values of $\log [\mathrm{H} / \mathrm{He}]$ agreed within $2 \sigma$; we conclude that there is no significant discrepancy between the hydrogen abundance measured from the optical and ultraviolet data.

We briefly explored two phenomena that are known to affect ultraviolet spectra of white dwarfs. Gänsicke et al. (2018) have shown that broadening of Ly $\alpha$ by neutral helium depends strongly on both $T_{\text {eff }}$ and $\log [\mathrm{H} / \mathrm{He}]$, and can result in asymmetric Ly $\alpha$ line profiles. As the fit of the blue 
wing of the Ly $\alpha(1140-1200 \AA)$ is noticeably poor in our analysis we investigated this feature with a small grid of models, including this broadening feature, around the bestfitting parameters. We fitted $T_{\text {eff }}$ and $\log [\mathrm{H} / \mathrm{He}]$ for three fixed values of $\log g(8.00,8.04,8.08$ : the canonical, spectroscopic fit, and photometric fit values), obtaining results that did not differ significantly from our determined values. From a visual inspection these improved the fit in the blue wing of Ly $\alpha$, but overall produced a worse FUV model. Therefore, we excluded this feature in our best-fitting model.

Pure hydrogen atmosphere white dwarfs with $T_{\text {eff }} \lesssim$ $20000 \mathrm{~K}$ exhibit a broad absorption feature near $1400 \AA$, which grows in strength as $T_{\text {eff }}$ decreases (Greenstein 1980; Wegner 1982, 1984). This has been identified as Ly $\alpha$ absorption caused by the $\mathrm{H}_{2}^{+}$quasi-molecule (Koester et al. 1985; Nelan \& Wegner 1985). A visual inspection suggested that this feature is not present in our COS spectrum. Nonetheless, given the unusually high abundance of hydrogen, we experimented with including this feature in our models, but did not achieve an improved fit. We conclude that, despite the ultraviolet and optical spectra being dominated by hydrogen, the $\mathrm{H}_{2}^{+}$feature does not form in the helium-dominated atmosphere of WD J2047-1259.

\subsection{Metal abundances}

We identified photospheric absorption lines from nine metals: carbon, oxygen, magnesium, silicon, phosphorus, sulphur, calcium, iron, and nickel (wavelengths in Table 3). We fitted these lines by iteratively adjusting the abundances until a visual best fit was found for both the ultraviolet and optical spectra. Uncertainty estimates are made from the spread of individual line strengths and the change in abundance required after a $\pm 1 \sigma$ adjustment in $T_{\text {eff }}$ and/or $\log g$. We also determine upper limits on the abundances of aluminium and nitrogen (Table 3.2).

In order to fit the narrow absorption features accurately, synthetic spectra must be convolved with an empirical line profile that reproduces instrumental broadening of narrow lines. Owing to the gradual degradation of the COS instrument, the line spread function (LSF) has become non-uniform and non-Gaussian. Characterisations of these functions are available online ${ }^{6}$. We interpolate a LSF for the wavelengths corresponding to each pixel and convolve these with the model spectra. This allows a better fit to absorption line profiles, particularly where adjacent lines are blended together. Absorption lines in the X-shooter spectra were convolved with a uniform Gaussian profile based on the instrumental characteristics ${ }^{7}$.

Close inspection of Fig. $1 \& 2$ reveals that not all absorption lines are well reproduced by the best-fitting model. For example, although the broader absorption features at $\simeq 1260 \AA$ are well fit, some narrower Si II lines in the ultraviolet are too strong in the model, while some optical absorption features appear slightly too weak. Some lines are blended with resonant absorption from the ISM (Mauche et al. 1988). This variable quality of fit - in particular for iron lines - reflects the varying accuracy and completeness

6 www.stsci.edu/hst/cos/performance/spectral_resolution/ 7 ww. eso.org/sci/facilities/paranal/instruments.html
Table 3. Wavelengths of absorption lines used to determine the relative abundances of each element. Those in italics are resonant ISM absorption lines.

\begin{tabular}{|c|c|}
\hline Ion & Vacuum Wavelengths $(\AA)$ \\
\hline $\mathrm{C}_{\mathrm{I}}$ & $\begin{array}{l}1140.35,1277.55,1328.83,1329.09,1329.10, \\
1329.58,1329.60\end{array}$ \\
\hline $\mathrm{C}$ II & $1323.91,1323.95,1334.53,1335.66,1335.71$ \\
\hline O I & $\begin{array}{l}1152.15,1302.17,1304.86,1306.03,7774.08 \\
7776.30,7777.53,8448.68\end{array}$ \\
\hline N I & $\begin{array}{l}1134.17,1134.41,1134.98,1167.45,1168.54 \\
1176.51,1199.55,1200.22,1200.71,1243.18 \\
1243.31,1310.54,1319.68,1411.95\end{array}$ \\
\hline Mg II & $4482.38,4482.58,7898.54,9220.78$ \\
\hline Al II & $\begin{array}{l}\text { 1189.19, } 1190.05,1191.81,3587.58,3901.78, \\
4664.35,5594.85,7044.03, \\
7058.66,7473.47\end{array}$ \\
\hline Al III & $1379.67,1384.13$ \\
\hline Si II & $\begin{array}{l}\text { 1190.42, 1193.29, 1194.50, 1197.39, 1246.74, } \\
\text { 1248.43, 1250.09, 1250.44, 1251.16, 1260.42, } \\
\text { 1264.74, 1265.00, 1304.37, 1305.59, 1309.28, } \\
\text { 1309.45, 1346.88, 1348.54, 1350.07, 1350.52, } \\
\text { 1350.66, 1352.64, 1353.72, 3857.11,4129.22, } \\
4132.06,5057.39,6348.86,6373.13\end{array}$ \\
\hline Si III & $\begin{array}{l}\text { 1108.36, 1109.94, 1109.97, 1113.17, 1113.20, } \\
\text { 1113.23, 1294.55, 1296.73, 1298.89, 1298.95, } \\
1301.15,1303.32,1312.59,1417.24\end{array}$ \\
\hline $\mathrm{P}_{\text {II }}$ & $1149.96,1152.82,1154.00,1155.01,1249.83$ \\
\hline S I & $1316.54,1425.03,1425.19$ \\
\hline S II & $\begin{array}{l}1124.40,1124.99,1131.06,1131.66,1250.58, \\
1253.81,1259.52\end{array}$ \\
\hline Ca II & $3180.25,3737.97,3934.78,3969.59,8544.44$ \\
\hline Fe II & $\begin{array}{l}1126.42,1126.59,1126.88,1142.31,1142.36, \\
1144.94,1147.41,1151.16,1262.14,1266.53, \\
1266.68,1267.42,1275.14,1275.78,1311.06, \\
1359.06,1361.37,1364.59,1364.76,1371.02, \\
1374.83,1374.94,1375.17,1379.47,1392.82, \\
1408.48,3228.67,5019.84,5170.47,\end{array}$ \\
\hline Fe III & $\begin{array}{l}1122.52,1124.87,1126.73,1128.04,1128.72, \\
1130.40,1131.19,1386.15\end{array}$ \\
\hline $\mathrm{Ni}$ II & $\begin{array}{l}1119.33,1133.73,1137.09,1139.64,1154.42, \\
1164.28,1164.58,1171.29,1173.30,1317.22, \\
1335.20,1345.88,1370.13,1374.07,1381.29, \\
1381.69,1393.32\end{array}$ \\
\hline
\end{tabular}

\begin{tabular}{lccrr}
\hline Element & $\log [\mathrm{Z} / \mathrm{He}]$ & $\begin{array}{c}\tau_{\text {diff }} \\
{\left[10^{3} \mathrm{yr}\right]}\end{array}$ & $\begin{array}{r}\text { Diffusion Flux } \\
{\left[10^{6} \mathrm{~g} \mathrm{~s}^{-1}\right]}\end{array}$ & $\begin{array}{r}X_{\mathrm{cvz}} \\
{\left[10^{18} \mathrm{~g}\right]}\end{array}$ \\
\hline $\mathrm{C}$ & $-6.1 \pm 0.1$ & 8.6 & 13 & 3.4 \\
$\mathrm{~N}$ & $<-7.0$ & 7.7 & - & - \\
$\mathrm{O}$ & $-4.8 \pm 0.1$ & 7.0 & 410 & 91.6 \\
$\mathrm{Mg}$ & $-5.6 \pm 0.1$ & 5.1 & 140 & 22.0 \\
$\mathrm{Al}$ & $<-6.5$ & 4.7 & - & - \\
$\mathrm{Si}$ & $-5.6 \pm 0.1$ & 4.7 & 170 & 25.5 \\
$\mathrm{P}$ & $-8.1 \pm 0.1$ & 4.2 & 1.1 & 0.1 \\
$\mathrm{~S}$ & $-6.6 \pm 0.1$ & 4.2 & 22 & 2.9 \\
$\mathrm{Ca}$ & $-6.9 \pm 0.1$ & 3.5 & 16 & 1.8 \\
$\mathrm{Fe}$ & $-6.4 \pm 0.2$ & 2.6 & 98 & 8.0 \\
$\mathrm{Ni}$ & $-7.4 \pm 0.1$ & 2.5 & 11 & 0.8 \\
\hline Total & & & 881 & 157 \\
\hline
\end{tabular}

in measurements of atomic data which confirm or replace theoretical calculations. These data are essential to much of the underlying work in astrophysics and must be supported by the astronomical community (Nave et al. 2019). 


\section{COMPOSITION OF ACCRETED MATERIAL}

The abundances of metals in a white dwarf's photosphere are a result of the composition of the parent body, the duration of the accretion event, and the rate at which each element gravitationally settles below the photosphere. Koester (2009, eq. 4) described the time-dependent convection zone mass $X_{\mathrm{cvz}}$ of a particular element, $Z$, which depends on each element's diffusion timescale, $\tau_{\text {diff }}$. The relative accretion rate of each metal $\dot{M}(Z, t)$ is assumed to be proportional to its relative abundance in the parent body. Thus, with measured photospheric abundances and computed diffusion timescales, one can determine the bulk composition of the parent body.

It is important to note that 3D atmospheric models are needed to truly understand the mixing processes in white dwarfs; Cukanovaite et al. $(2018,2019)$ have made significant progress, but are yet to extend to atmospheric compositions like that of WD J2047-1259. Similarly, Bauer \& Bildsten (2018) showed that thermohaline mixing in hydrogen atmospheres can change the accretion rates by orders of magnitude, and to some extent the inferred compositions, but no equivalent study has been published for helium atmospheres. Thus, for the remainder of this work, we take the conventional approach based on static, one-dimensional envelope models with homogeneous abundance profiles: Koester (2009) examined a simplified accretion episode wherein the accretion rate is described by a step function; switching on at $t=0$, and then falling instantaneously to zero after some finite duration. This results in three key phases of an accretion episode, for which we adopt the clearer terminology from Swan et al. (2019b):

- Increasing Phase: when accretion begins, the rate of gravitational settling is initially negligible. Metal abundances in the atmosphere increase linearly with time, and abundance ratios are equal to those of the parent body. This strictly holds only while $t \ll \tau_{\text {diff }}$, but is a reasonable approximation up to $t<\tau_{\text {diff }}$.

- Steady State: after $\simeq 5$ diffusion timescales have passed, accretion and diffusion reach a balanced state where atmospheric abundances are constant. Relative abundances must be multiplied by the ratio of element diffusion timescales to recover the ratios in the parent body. This phase lasts for as long as accretion is ongoing.

- Decreasing Phase: metal abundances in the photosphere exponentially decay on their individual diffusion timescales. Atmospheric abundance ratios diverge from those of the parent body. Absorption lines can remain detectable for several diffusion timescales after accretion stops.

Using the photospheric metal abundances and diffusion timescales listed in Table 3.2, we calculated the parent body abundances - relative to silicon - for each phase. For the decreasing phase, the abundances depend on the assumed interval since the abrupt end of accretion; we adopt $2 \tau_{\text {diff }}(\mathrm{Si})$ (9320) yr. These abundances, ordered by atomic mass, are shown in Fig. 4, shown with elements ordered by condensation temperature.

The increasing and steady state abundances are similar; both are rich in carbon, sulphur, and oxygen, but poor in iron and nickel, relative to the bulk Earth. The abundances suggest WD J2047-1259 is accreting the debris of a rocky planetesimal broadly similar to carbonaceous chondrite (C chondrite) material (Kallemeyn \& Wasson 1981; Wasson \& Kallemeyn 1988; Lodders 2003, 2010), most well matched to the CM chondrite subgroup ${ }^{8}$. Iron and nickel abundances are both well below bulk Earth levels, and slightly below the two illustrated classes of $\mathrm{C}$ chondrite. Within the uncertainties of our measurements, and the diversity among Solar-system chondrites, the abundances of the planetesimal accreted by WD J2047-1259 suggest that it had not undergone major alterations in the form of melting or differentiation.

The increasing phase can last for $\sim \tau_{\text {diff }}$ (in this case, $\sim 10^{4} \mathrm{yr}$ ), whereas the steady state phase is estimated to last for $10^{5.6 \pm 1.1} \mathrm{yr}$, (Girven et al. 2012, based on estimated lifetimes of debris discs). The increasing phase does not produce a significantly favourable composition - the abundances of iron and nickel are better reproduced by the steady state phase. Thus, we favour the steady state phase composition.

Adopting that WD J2047-1259 is in the decreasing phase brings the iron and nickel abundances in line with those of the bulk Earth, but leaves the volatiles carbon and sulphur highly over-abundant. Wilson et al. (2016) have shown that the $\mathrm{C} / \mathrm{O}$ abundance ratio can distinguish between $\mathrm{C}$ chondrite and bulk Earth compositions (for which $\log [\mathrm{C} / \mathrm{O}] \simeq-1$ (Wasson \& Kallemeyn 1988; Lodders 2010), and $-1.8--2.7$ (Allègre et al. 2001), respectively). The $\mathrm{C} / \mathrm{O}$ ratio is not strongly affected by the assumptions about the accretion history, as both elements have relatively similar sinking times (Table 3.2 ): We calculate $\log [\mathrm{C} / \mathrm{O}]=-1.30,-1.39$, and -1.50 for the increasing phase, steady state, and decreasing phase, respectively, with uncertainties of \pm 0.14 . Hence, the C/O ratio of WD J2047-1259 alone indicates that the accreted material is similar to Solar System C chondrites, and enhanced compared to bulk Earth. We conclude that the abundances implied by assuming WD J2047-1259 is in the decreasing phase do not match bulk Earth, or other Solar System compositions known to us.

The convection zone of WD J2047-1259 has a mass fraction of $\log q=\log \left(M_{\mathrm{cvz}} / M_{*}\right)=-8.92$ (based on Koester 2009). From the measured abundances, we determine $1.57 \times 10^{20} \mathrm{~g}$ of metals in the convection zone. A spherical object of this mass, and a density of $3 \mathrm{~g} \mathrm{~cm}^{-3}$, would have a radius of $\simeq 23 \mathrm{~km}$; slightly smaller than Mathilde - incidentally a Ctype asteroid rich in carbon (Yeomans et al. 1997). This is a lower limit on the size of the object. For the steady state phase to maintain this mass requires a diffusion flux of $8.8 \times 10^{8} \mathrm{~g} \mathrm{~s}^{-1}$, which is by definition equal to the accretion rate $\dot{M}_{\text {acc }}$. This value is typical of the measured accretion rates for polluted white dwarfs of similar temperatures, although helium-dominated white dwarfs show a large scatter, extending to higher accretion rates (Koester \& Wilken 2006; Farihi et al. 2012b, see also fig. 4. of Bauer \& Bildsten 2018).

The water content of the accreted material can be estimated by calculating the oxygen budget (first explored by Klein et al. 2010); comparing the abundance of oxygen with the abundances of elements that are in common minerals. The assumption that the geochemistry of extra-solar rocky

$8 \mathrm{CM}$ chondrites are the most commonly found type of $\mathrm{C}$ chondrites, named after the Mighei meteorite. Subclasses of $\mathrm{C}$ chondrites are distinguished by chemical and petrological properties. 


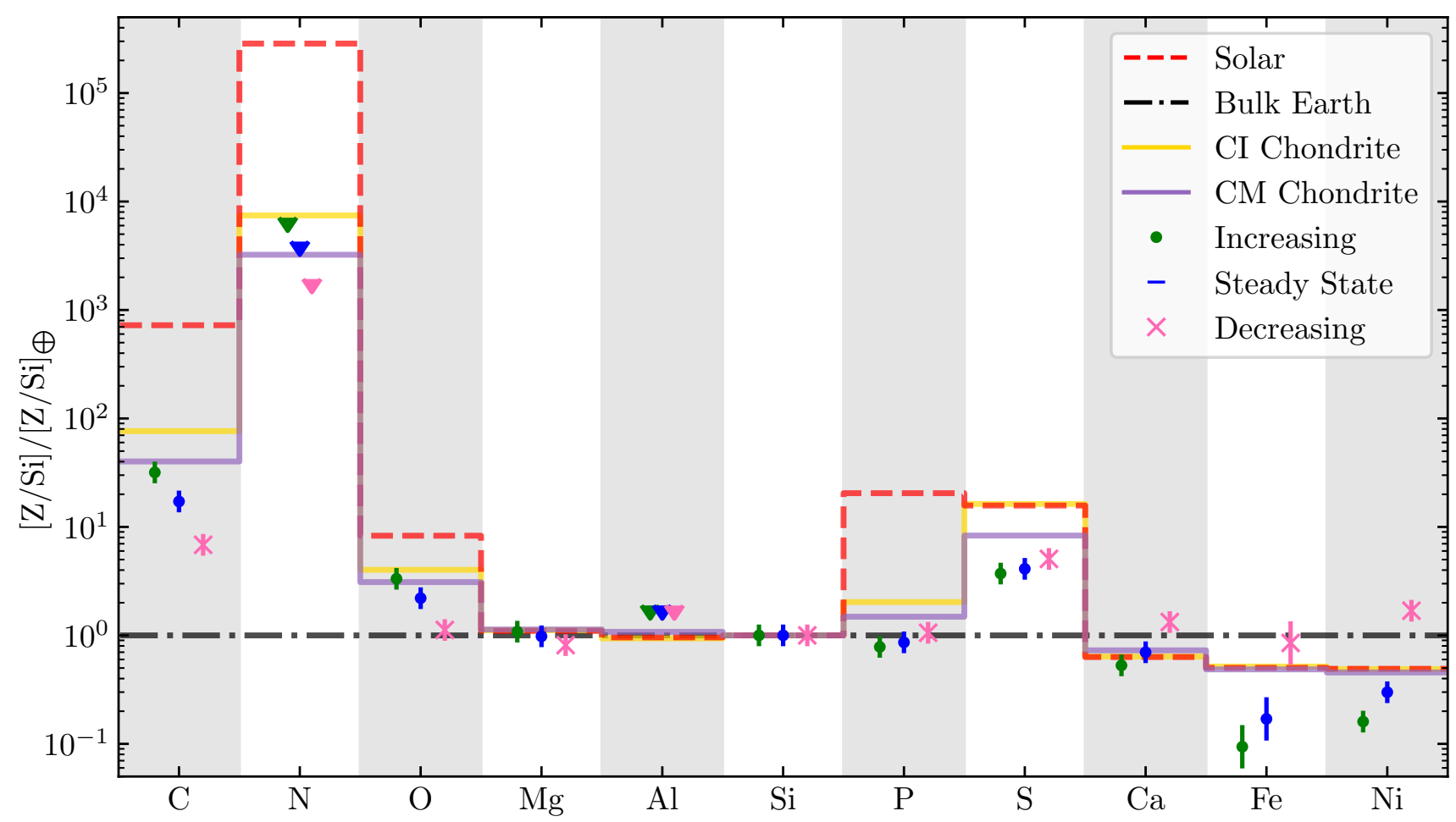

Figure 4. Abundances of metals, relative to silicon, in the parent body, normalised to the corresponding bulk Earth ratio, and calculated for each accretion phase. The decreasing phase is shown for $2 \tau_{\text {diff }}(\mathrm{Si})$ after accretion ends. Chondritic compositions show the closest match to the abundances of most of the measured elements; carbon, oxygen and sulphur are best matched to CM chondrite composition. Upper limits are shown by triangles.

bodies resembles that of the Solar System was demonstrated by Doyle et al. (2019). In the bulk Earth, almost all oxygen is in the metal-oxides common to rocky material; principally $\mathrm{SiO}_{2}, \mathrm{MgO}$ and $\mathrm{FeO}$, with smaller contributions from other minerals (e.g. $\mathrm{Al}_{2} \mathrm{O}_{3}, \mathrm{CaO}$ ), and a negligible amount of water. In planetesimals formed beyond the snowline, a large fraction of the oxygen budget comes from water.

Using the compositions determined for each accretion phase, we examine the oxygen budget in the accreted material. We use Monte Carlo methods to sample the number of atoms of each mineral forming element in our simulated composition. These are multiplied by the empirical ratio for the chemical compound ( $\frac{y}{x}$ for $\mathrm{Z}_{x} \mathrm{O}_{y}$; e.g. $\frac{2}{1}$ for $\left.\mathrm{SiO}_{2}\right)$, summed, and then compared to the total number of oxygen atoms in the simulation. For rocky material devoid of water, these numbers should be equal; i.e. 100 per cent of the oxygen budget is used. A median less than 100 per cent indicates an oxygen excess, best explained by water in the parent body. If the median is beyond 100 per cent, some fraction of the parent body would be metallic. This is not uncommon for iron and nickel, but a metallic component including other mineral-forming elements is unlike any known Solar System body. Doyle et al. (2019) have shown that the chemistry resulting from rock formation in exo-planetary systems is broadly similar to that of the Solar System.

As we only have an upper limit on the abundance of aluminum, $\log [\mathrm{Al} / \mathrm{He}]<-6.5$, we included it in the analysis at its expected abundance for bulk Earth and C chondrite compo- sitions; $\log [\mathrm{Al} / \mathrm{Si}]=-1.05$ (e.g. Lodders 2010 ), but with zero uncertainty. Not accounting for aluminium would cause a significant systematic offset in the oxygen budget, and our adopted approach is robust as the abundance of aluminium does not vary significantly between bulk Earth and chondritic compositions.

The resulting distributions are approximately lognormal. In the increasing and steady state phases (Fig. 5a) we find an oxygen excess, $\iota$, of $46_{-17}^{+13}$ and $16_{-27}^{+20}$ per cent, respectively (uncertainties are from the $16^{\text {th }}$ and $84^{\text {th }}$ percentiles of the distributions). The corresponding median mass fractions of water in the accreted material are 29 and 8 per cent. $\mathrm{C}$ chondrite meteorite samples typically contain $10-25$ per cent water by mass, varying slightly between subgroups (Garenne et al. 2014). We find excess oxygen in 98 and 74 per cent of simulated compositions, respectively.

Jura \& Xu (2010) suggested that survival of water through the AGB phase is limited to that sequestered tens of kilometres within a rocky body. For example, Malamud \& Perets $(2016,2017 \mathrm{a}, \mathrm{b})$ predict that $\mathrm{a} \simeq 50 \mathrm{~km}$ minor planet formed at $\simeq 40 \mathrm{AU}$ around a $2 \mathrm{M}_{\odot}$ progenitor (evolving into a $0.59 \mathrm{M}_{\odot}$ white dwarf) would retain half of its water through stellar evolution. The eccentric Kozai-Lidov mechanism has been shown to cause instabilities in wide orbit planetesimals large enough to cause their accretion (Stephan et al. 2017).

For the decreasing phase (Fig. 5b) we show the oxygen budget at $2 \tau_{\text {diff }}(\mathrm{Si})$ after accretion ends, for two different mineralogies; rocky, in which all rock-forming elements are 


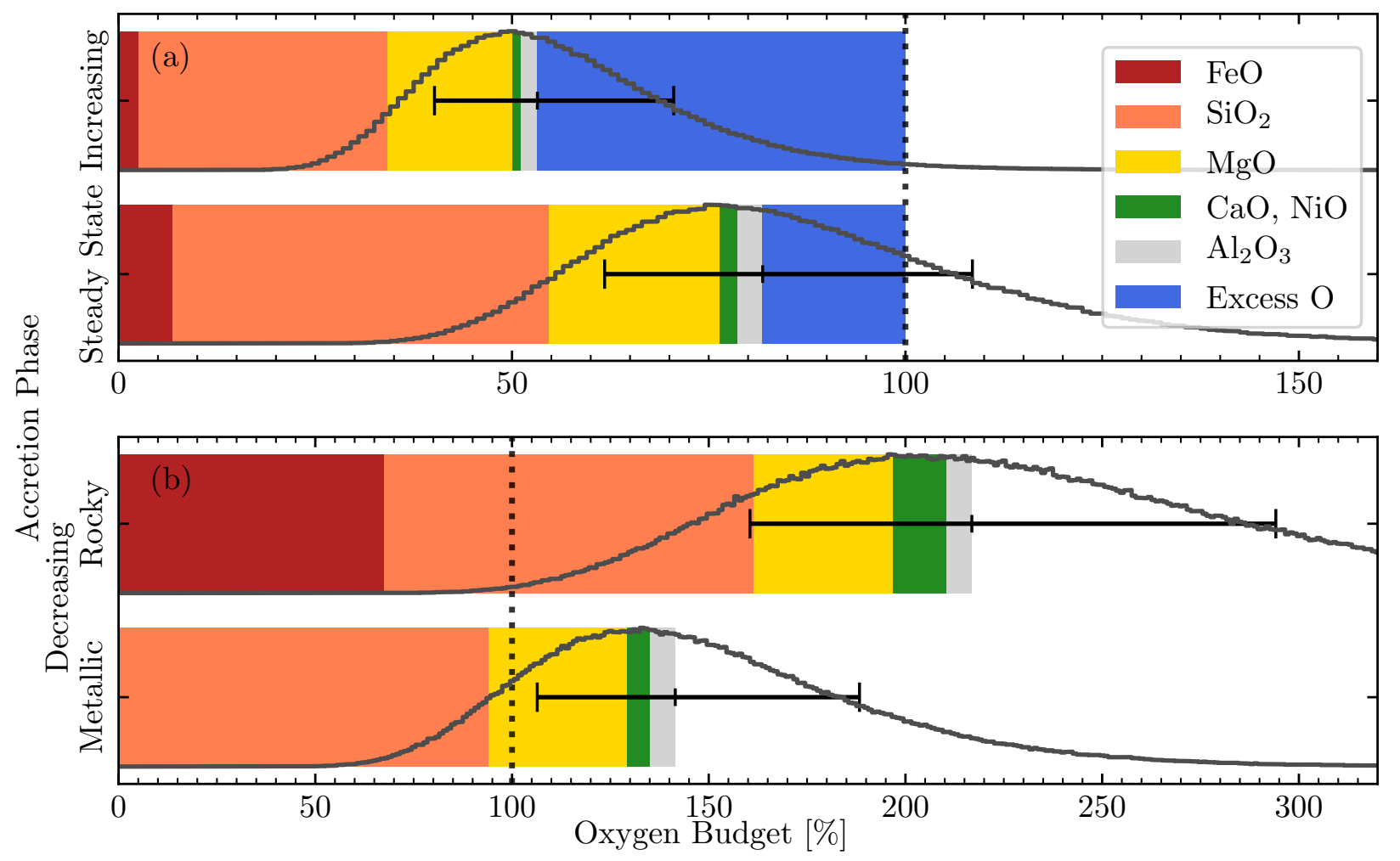

Figure 5. The results of oxygen budget simulations for (a) increasing and steady state phases; (b) decreasing phase, $2 \tau_{\text {diff }}(\mathrm{Si})$ after accretion ends, for a completely rocky parent body and for one with metallic iron and nickel. NB: Axis scales differ between (a) and (b). Solid lines show the distribution of oxygen budget usage in our simulations, for each phase: A higher percentage indicates a relative paucity of oxygen compared to the abundances of mineral-forming elements. The error bars mark the $50 \pm 34.1$ percentiles of the distributions. The coloured bars show the breakdown of the median oxygen budget by minerals.

oxidised, and metallic, where iron and nickel are in their metallic form and do not affect the oxygen budget. Other common oxidation states (e.g. $\mathrm{Fe}_{2} \mathrm{O}_{3}$ and $\mathrm{NiO}_{2}$ ) for iron and nickel are not investigated. The oxidation state of iron has been shown to vary across subgroups of $\mathrm{C}$ chondrites, but always some iron is in an oxidised state (Garenne et al. 2019). The oxidation state of the parent body accreted by WD J2047-1259 may lie between our simplified rocky and metallic cases. For both cases, the result is an oxygen deficit.

We calculated a parent body composition, and subsequent oxygen budget, for a range of times after the end of accretion, to find when the computed abundances become unrealistic. We accounted for wholly rocky mineralogy, and for a parent body with purely metallic iron and nickel. The constraints on the maximum duration of the decreasing phase are given in Table 4 . These are the times at which the computed composition distributions indicate an oxygen deficit at the $95^{\text {th }}$ and $99.7^{\text {th }}$ percentiles (two and three sigma confidence). These upper limits are nearly two orders of magnitude shorter than estimated disc lifetimes of $10^{5.6 \pm 1.1} \mathrm{yr}$ (Girven et al. 2012), although within two sigma. We interpret this, in combination with the $\mathrm{C} / \mathrm{O}$ ratio mentioned above, as evidence that the decreasing phase scenario is unlikely to be correct for WD J2047-1259.

We next consider the possible mass of water delivered to WD J2047-1259, $M_{\mathrm{H}_{2} \mathrm{O}}$, adopting the steady state phase and the median oxygen excess value. Assuming at least five
Table 4. Time constraints on the decreasing phase for different mineralogies $\left(\tau_{\mathrm{Si}}=4660 \mathrm{yr}\right)$.

\begin{tabular}{lcc}
\hline & $95^{\text {th }}$ percentile & $99.7^{\text {th }}$ percentile \\
& \multicolumn{2}{c}{$\left[\tau_{\text {diff }}(\mathrm{Si})\right]$} \\
\hline Metallic $\mathrm{Fe} \& \mathrm{Ni}$ & 2.32 & 3.23 \\
$\mathrm{FeO} \mathrm{\&} \mathrm{NiO}$ & 1.40 & 1.78 \\
\hline
\end{tabular}

diffusion timescales have passed since the start of the accretion episode (in order to reach the steady state), we derive $M_{\mathrm{H}_{2} \mathrm{O}} \geq 5 \tau_{\text {diff }}(\mathrm{Si}) \times \dot{M}_{\text {acc }} \times \iota=5 \times 10^{19} \mathrm{~g}$. This is a small amount compared to that held by some Solar System objects - four orders of magnitude less than that in Ceres $\left(\sim 10^{23} \mathrm{~g}\right)$. We discuss the possible implications of water accretion, and the total hydrogen content in the atmosphere of WD J2047-1259, further in Section 6.

\section{NO INFRARED EXCESS}

Coverage of infrared wavelengths is provided by archival Spitzer data (Fig. 3), and the Near Infrared (NIR) X-shooter spectrum. The Spitzer photometric data lie within $\pm 1 \sigma$ of synthetic photometry for the featureless blackbody of the same temperature. The NIR spectrum is consistent with this evidence, and Fig. 3 shows it to be compatible with the Rayleigh-Jeans tail of the atmospheric model.

As we find a steady state accretion phase the most likely, 
the presence of an accretion disc is implied - but is not confirmed by these data. Among single white dwarfs, metal pollution is detected far more frequently than warm debris discs (Wilson et al. 2019) . Disc detections correlate with high accretion rates, typically $\dot{M}_{\text {acc }} \gtrsim 10^{9} \mathrm{~g} \mathrm{~s}^{-1}$ (Bergfors et al. 2014). The accretion rate for WD J2047-1259 is slightly lower $8.8 \times 10^{8} \mathrm{~g} \mathrm{~s}^{-1}-$ so a non-detection is not unusual for this system; indeed there are multiple examples of white dwarfs that must be accreting material, but have no infrared excess detection. For example, a thin edge-on disc could be the cause of this non-detection (Bonsor et al. 2017).

\section{HYDROGEN IN HELIUM ATMOSPHERES}

WD J2047-1259 is the latest addition to the small group of peculiar white dwarfs with helium-dominated atmospheres that show anomalously large hydrogen abundances and photospheric metal pollution from accreted planetary material. These appear to be the extreme outliers from the large population of helium-dominated white dwarfs thought to be accreting hydrogen and metals from water-bearing rocky material (Gentile Fusillo et al. 2017).

\subsection{The argument for water accretion}

Rolland et al. (2018) measured the hydrogen abundances of a sample of 143 helium rich white dwarfs, the majority of which contain more hydrogen than can be explained by the scenarios they explore. Rolland et al. (2018) initially used models that were chemically homogeneous, but Rolland et al. (2020) have since implemented more complex abundance profile inputs. The authors modeled white dwarfs containing a fixed mass of hydrogen, wherein two possibilities arise: If the initial hydrogen mass is sufficiently large $\left(\gtrsim 10^{-14} \mathrm{M}_{\odot}\right)$, once the white dwarf has cooled to $\simeq 25000 \mathrm{~K}$ its envelope becomes stratified as hydrogen floats to the surface, and consequently helium absorption lines are no longer detectable as the photosphere is within the hydrogen layer. For lower initial hydrogen masses no stratification occurs, but hydrogen is diluted as the convective layer grows deeper, and eventually becomes undetectable. The hydrogen masses, measured from observations of helium-rich white dwarfs (Koester \& Kepler 2015; Rolland et al. 2018), in the range $12000-20000 \mathrm{~K}$ cannot have been present throughout the history of those stars, or they would have become stratified and remained so.

Seeking an alternative explanation, Rolland et al. (2018, 2020) examined white dwarfs with negligible initial hydrogen mass, but which accrete hydrogen at a constant rate. The resulting picture is similar: if this accretion rate is too high $\left(\gtrsim 10^{-23} \mathrm{M}_{\odot} \mathrm{yr}^{-1} \simeq 10^{3} \mathrm{~g} \mathrm{~s}^{-1}\right)$, the envelope inevitably becomes stratified as too much hydrogen will be accrued before the convection zone develops, which is subsequently suppressed. For lower accretion rates, hydrogen remains well mixed and dilutes as the convection zone grows, but the mass of hydrogen accrued cannot produce the observed abundances in the range 12000-20000 K. Rolland et al. (2018, 2020) concluded that the measured hydrogen masses could not be explained by left over hydrogen from the progenitor, nor by a constant rate of accretion. Rolland et al. (2020) also sketched out a novel scenario in which hydrogen can be dredged up from deep within the envelope, which has potential to explain a larger portion of the observations.

An accretion scenario will only work if the start of accretion is delayed until when the convection zone begins to grow significantly, at $\simeq 24000 \mathrm{~K}$ (fig. 2 of Rolland et al. 2020) Dynamical simulations have found that planetesimals are not scattered to within the Roche radius of the white dwarf for the first $\simeq 40 \mathrm{Myr}$ of its cooling lifetime (Mustill et al. 2018 ) - corresponding to $T_{\text {eff }} \simeq 23000 \mathrm{~K}$ for a $0.6 \mathrm{M}_{\odot}$ white dwarf. These simulations show that subsequent Roche limit crossings occurred stochastically. The delay in the onset of accretion demonstrates that the varying accretion history may provide a natural explanation for the delivery of (waterrich) planetary debris after white dwarfs have developed sufficiently deep convection zones that prevent stratified atmospheric configurations.

\subsection{The specific case of WDJ2047-1259}

Here, we consider the plausible hydrogen accretion histories for WD J2047-1259. We have found a water mass fraction of approximately eight per cent in the accreted material from the median oxygen excess in our steady state composition simulations. Based on the measured abundance of hydrogen and envelope mass from our model, the total mass of hydrogen present in the atmosphere is $M_{\mathrm{H}}=3 \times 10^{22} \mathrm{~g}$ $\left(\sim 10^{-11} \mathrm{M}_{\odot}\right)$; orders of magnitude above the limits imposed on the initial hydrogen mass $M_{\mathrm{H}} \lesssim 10^{-14} \mathrm{M}_{\odot}$ for the envelope not to become stratified (Rolland et al. 2018, 2020); according to fig. 4 of Rolland et al. (2020) a star with this $T_{\text {eff }}$ and $M_{\mathrm{H}}$ should be stratified.

We begin with the case in which a single body delivered all of the accumulated hydrogen during the current accretion episode. Assuming no change in the composition or rate of accretion, the parent body mass required is $\sim 10^{24} \mathrm{~g}$; comparable to the mass of Ceres (albeit with a lower water fraction). The current episode would have to have lasted for approximately $115 \mathrm{Myr}$ - most of the cooling age of the star (125 Myr). Given the stochastic accretion predicted by Mustill et al. (2018), and disc lifetime estimates, which range between 0.01-10 Myr, (Jura 2008; Metzger et al. 2012; Girven et al. 2012), the hypothesis of a single parent body dominating accretion onto WD J2047-1259 for 100 Myr is unreasonable. We exclude the possibility that WD J20471259 has undergone monotonous accretion from a single object for $>10 \mathrm{Myr}$. Further, accretion spanning most of the cooling age of the star would require an average rate of $\sim 10^{-19} \mathrm{M}_{\odot} \mathrm{yr}^{-1}$ to accumulate this mass of hydrogen. This rate is three orders of magnitude above the limit imposed by Rolland et al. (2018, 2020, see fig. 8 of the latter).

The only plausible explanation involving a single body of this size is that the accretion rate has previously fluctuated to much higher values, such that the duration of the episode could be $\lesssim 10 \mathrm{Myr}$. If the rate had been orders of magnitude higher in the last several diffusion timescales $\left(\sim 10^{4} \mathrm{yr}\right)$ we would see evidence in the abundance ratios. The details of the accretion process from circumstellar debris discs are not well understood, but variable infrared emission has been seen in many systems (Xu \& Jura 2014; Xu et al. 2018; Farihi et al. 2018; Swan et al. 2019a; Wang et al. 2019) and calcium emission line strength variations also suggest rapid changes (relative to the disc lifetime) 
within the gaseous component of debris discs (Wilson et al. 2014; Manser et al. 2019). However, these have not been directly linked to changes in the rate of accretion. To date, there are no observations showing a changing accretion rate at a white dwarf.

Given our understanding of remnant planetary systems, it is almost certain that WD J2047-1259 has accreted planetesimals in the past. The resulting metal pollution may have long-since sunk out of the photosphere and become undetectable. However, hydrogen may have been added to the photosphere by any of these past events. Therefore we consider a scenario wherein the current accretion episode is the latest in a stochastic series over the last $\simeq 85 \mathrm{Myr}$ (for accretion beginning $\simeq 40 \mathrm{Myr}$ into the cooling lifetime, as suggested by Mustill et al. 2018), during which most of the current hydrogen must have been accreted. This is compatible with fig. 9 of Mustill et al. (2018) which shows that planetesimal Roche lobe crossings become roughly constant (though still stochastic) at a cooling age of $60 \mathrm{Myr}$. For a canonical white dwarf, $60 \mathrm{Myr}$ corresponds to a temperature of $\simeq 21000 \mathrm{~K}$. According to Rolland et al. (2020, fig. 4), the maximum permitted hydrogen mass at this temperature, whilst retaining a helium-dominate photosphere, is $\sim 10^{-14} \mathrm{M}_{\odot}$; three orders of magnitude below the current mass in WD J2047-1259. We thus conclude that 99.9 per cent of the hydrogen present in WD J2047-1259 has been delivered by accretion within the last $60 \mathrm{Myr}$. The large amount of water delivered (compared to other helium atmosphere white dwarfs of similar temperatures) in this system may be attributed to some unusual architecture of the surviving planetary system, or an abundance of water far in excess of the typical planetary systems (Jura \& Xu 2012; Gentile Fusillo et al. 2017).

\section{CONCLUSIONS}

We have characterised of the atmosphere of WD J20471259 which contains $1.6 \times 10^{20} \mathrm{~g}$ of metals; carbon, oxygen, magnesium, silicon, phosphorus, sulphur, calcium, iron, and nickel. We also confirm the previously reported large hydrogen abundance in the helium dominated atmosphere, finding $T_{\text {eff }}=17,970 \pm{ }_{170}^{140} \mathrm{~K}, \log g=8.04 \pm 0.05$ and $\log [\mathrm{H} / \mathrm{He}]=$ $-1.08 \pm 0.08$. We show that the photosphere is polluted by rocky material rich in volatile and transitional elements compared to bulk Earth, but consistent with $\mathrm{C}$ chondrites (in particular, most similar to the CM chondrites). It is most likely that accretion is in steady state, and that the parent body probably contained a small amount water; we find excess oxygen in 74 per cent of simulated steady state compositions, resulting in a median eight per cent water by mass.

Neither the increasing nor decreasing phase can be definitively excluded, but both are subject to severe constraints to their duration in comparison to the steady state phase. The increasing phase scenario yields a much higher water fraction by mass in the accreted material (29 per cent), and a composition that closely resembles $\mathrm{C}$ chondrites. The decreasing phase scenario yields a composition unlike any known planetary body in the Solar System, as well as an implausible oxygen budget.

We infer $3 \times 10^{22} \mathrm{~g}$ of hydrogen in the atmosphere of this star. Although convective mixing processes may have altered the atmosphere of WD J2047-1259, no such process can account for its current state. The star must have acquired its extraordinary abundance of hydrogen within the last $\simeq 60 \mathrm{Myr}$. We find only two plausible scenarios that could explain this:

- An ongoing accretion episode of an object with mass similar to (or larger than) Ceres. The accretion rate was previously significantly higher, in order that the accretion episode last $\leq 10 \mathrm{Myr}$, but must have been stable at the current level for several diffusion timescales $(\simeq 0.01 \mathrm{Myr})$.

- A stochastic series of accretion episodes, which began within the last $\simeq 85 \mathrm{Myr}$. Many of these episodes have delivered water-rich material to the white dwarf; within the last $\simeq 60 \mathrm{Myr} \sim 10^{-11} \mathrm{M}_{\odot}$ of hydrogen was accreted. This hydrogen has most likely come from a large number of waterbearing planetesimals (e.g chondrites) or from a small number of large objects that contain water.

The latter scenario is more consistent with our current understanding of how remnant planetary systems evolve around white dwarfs, and suggests that this exo-planetary system could be either particularly water rich, or currently has an architecture that perturbs an abnormally large number of water-bearing objects on wide orbits. If all of this hydrogen was delivered as water, it would equate to five per cent of Earth's oceans.

\section{ACKNOWLEDGEMENTS}

MJH is supported by the UK Science and Technology Facilities Council studentship ST/R505195/1, and thanks KMZ and JM for their encouragement during this work. OT was supported by a Leverhulme Trust Research Project Grant. The research leading to these results has received funding from the European Research Council under the European Union's Seventh Framework Programme (FP/2007-2013) / ERC Grant Agreement n. 320964 (WDTracer). CJM and BTG were supported by the UK STFC grant ST/P00049. $\mathrm{RR}$ acknowledges funding by the German Science foundation (DFG) through grants HE1356/71-1 and IR190/1-1, and funding from the postdoctoral fellowship programme Beatriu de Pinós, funded by the Secretary of Universities and Research (Government of Catalonia) and by the Horizon 2020 programme of research and innovation of the European Union under the Maria Skłodowska-Curie grant agreement No 801370. The research leading to these results has received funding from the European Research Council under the European Union's Horizon 2020 research and innovation programme no. 677706 (WD3D). AS acknowledges support from an STFC studentship.

This work has made use of data from the European Space Agency (ESA) mission Gaia (https://www.cosmos. esa.int/gaia), processed by the Gaia Data Processing and Analysis Consortium (DPAC, https://www.cosmos.esa. int/web/gaia/dpac/consortium). Funding for the DPAC has been provided by national institutions, in particular institutions participating in the Gaia Multilateral Agreement. This work is based on observations collected at the European Southern Observatory under ESO programme 0101.C0646, and on observations made with the NASA/ESA Hubble Space Telescope, obtained at the Space Telescope Science 
Institute, which is operated by the Association of Universities for Research in Astronomy, Inc., under NASA contract NAS 5-26555. These observations are associated with programmes \#15073 and \#15474. This publication makes use of data products from the Two Micron All Sky Survey, which is a joint project of the University of Massachusetts and the Infrared Processing and Analysis Center/California Institute of Technology, funded by the National Aeronautics and Space Administration and the National Science Foundation. The identification of individual lines made use of the National Institute of Standards and Technology Atomic Spectra Database Lines Form . The Pan-STARRS1 Surveys (PS1) and the PS1 public science archive have been made possible through contributions by the Institute for Astronomy, the University of Hawaii, the Pan-STARRS Project Office, the Max-Planck Society and its participating institutes, the Max Planck Institute for Astronomy, Heidelberg and the Max Planck Institute for Extraterrestrial Physics, Garching, The Johns Hopkins University, Durham University, the University of Edinburgh, the Queen's University Belfast, the Harvard-Smithsonian Center for Astrophysics, the Las Cumbres Observatory Global Telescope Network Incorporated, the National Central University of Taiwan, the Space Telescope Science Institute, the National Aeronautics and Space Administration under Grant No. NNX08AR22G issued through the Planetary Science Division of the NASA Science Mission Directorate, the National Science Foundation Grant No. AST-1238877, the University of Maryland, Eotvos Lorand University (ELTE), the Los Alamos National Laboratory, and the Gordon and Betty Moore Foundation.

\section{DATA AVAILABILITY}

Data underlying this article are available in the MAST archive at https://mast.stsci.edu/portal/Mashup/ Clients/Mast/Portal.html, and can be accessed with observation IDs ldnu0r010, ldst02010, and ldst02020.

Data underlying this article are available in the ESO Science Archive at http://archive.eso.org/ scienceportal/home, and can be accessed with program ID 0101.C-0646.

\section{REFERENCES}

Allègre C., Manhès G., Lewin É., 2001, EPSL, 185, 49

Althaus L. G., Córsico A. H., Isern J., García-Berro E., 2010, A\&ARv, 18, 471

Bauer E. B., Bildsten L., 2018, ApJ, 859, L19

Beauchamp A., Wesemael F., Bergeron P., Liebert J., Saffer R. A., 1996, in Jeffery C. S., Heber U., eds, Astronomical Society of the Pacific Conference Series Vol. 96, Hydrogen Deficient Stars. p. 295

Bergeron P., et al., 2011, ApJ, 737, 28

Bergfors C., Farihi J., Dufour P., Rocchetto M., 2014, MNRAS, 444,2147

Bianchi L., Herald J., Efremova B., Girardi L., Zabot A., Marigo P., Conti A., Shiao B., 2011, Ap\&SS, 335, 161

Bonsor A., Farihi J., Wyatt M. C., van Lieshout R., 2017, MNRAS, 468, 154

Brinkworth C. S., Gänsicke B. T., Girven J. M., Hoard D. W., Marsh T. R., Parsons S. G., Koester D., 2012, ApJ, 750, 86
Capitanio L., Lallement R., Vergely J. L., Elyajouri M., MonrealIbero A., 2017, A\&A, 606, A65

Chambers K. C., Pan-STARRS Team 2016, in American Astronomical Society Meeting Abstracts \#227. p. 324.07

Chayer P., Fontaine G., Wesemael F., 1995, AJ, 99, 189

Cukanovaite E., Tremblay P. E., Freytag B., Ludwig H. G., Bergeron P., 2018, MNRAS, 481, 1522

Cukanovaite E., Tremblay P. E., Freytag B., Ludwig H. G., Fontaine G., Brassard P., Toloza O., Koester D., 2019, MNRAS, p. 2282

Cunningham T., Tremblay P.-E., Gentile Fusillo N. P., Hollands M., Cukanovaite E., 2020, MNRAS, 492, 3540

Cutri R. M., et al., 2003, VizieR Online Data Catalog

Debes J. H., Hoard D. W., Wachter S., Leisawitz D. T., Cohen M., 2011, ApJS, 197, 38

Doyle A. E., Young E. D., Klein B., Zuckerman B., Schlichting H. E., 2019, Science, 366, 356

Eisenstein D. J., et al., 2006, AJ, 132, 676

Farihi J., 2016, New Astronomy Reviews, 71, 9

Farihi J., Gänsicke B. T., Steele P. R., Girven J., Burleigh M. R., Breedt E., Koester D., 2012a, MNRAS, 421, 1635

Farihi J., Gänsicke B. T., Wyatt M. C., Girven J., Pringle J. E., King A. R., 2012b, MNRAS, 424, 464

Farihi J., Gänsicke B. T., Koester D., 2013, Science, 342, 218

Farihi J., Koester D., Zuckerman B., Vican L., Gänsicke B. T., Smith N., Walth G., Breedt E., 2016, MNRAS, 463, 3186

Farihi J., et al., 2018, MNRAS, 481, 2601

Fitzpatrick E. L., 1999, PASP, 111, 63

Fontaine G., Wesemael F., 1987, in Philip A. G. D., Hayes D. S., Liebert J. W., eds, IAU Colloq. 95: Second Conference on Faint Blue Stars. pp 319-326

Fontaine G., Brassard P., Bergeron P., 2001, PASP, 113, 409

Freudling W., Romaniello M., Bramich D. M., Ballester P., Forchi V., García-Dabló C. E., Moehler S., Neeser M. J., 2013, A\&A, 559, A96

Gaia Collaboration et al., 2016, A\&A, 595, A1

Gänsicke B. T., Marsh T. R., Southworth J., Rebassa-Mansergas A., 2006, Science, 314, 1908

Gänsicke B. T., Marsh T. R., Southworth J., 2007, MNRAS, 380, L35

Gänsicke B. T., Koester D., Marsh T. R., Rebassa-Mansergas A., Southworth J., 2008, MNRAS, 391, L103

Gänsicke B. T., Koester D., Farihi J., Girven J., Parsons S. G., Breedt E., 2012, MNRAS, 424, 333

Gänsicke B. T., Koester D., Farihi J., Toloza O., 2018, MNRAS, p. 2415

Garenne A., Beck P., Montes-Hernandez G., Chiriac R., Toche F., Quirico E., Bonal L., Schmitt B., 2014, Geochimica Cosmochimica Acta, 137, 93

Garenne A., Beck P., Montes-Hernandez G., Bonal L., Quirico E., Proux O., Hazemann J. L., 2019, Meteoritics and Planetary Science, 54, 2652

Gentile Fusillo N. P., Gänsicke B. T., Farihi J., Koester D., Schreiber M. R., Pala A. F., 2017, MNRAS, 468, 971

Gentile Fusillo N. P., et al., 2019, MNRAS, 482, 4570

Girven J., Brinkworth C. S., Farihi J., Gänsicke B. T., Hoard D. W., Marsh T. R., Koester D., 2012, ApJ, 749, 154

Graham J. R., Matthews K., Neugebauer G., Soifer B. T., 1990, ApJ, 357, 216

Greenstein J. L., 1980, ApJ, 241, L89

Harrison J. H. D., Bonsor A., Madhusudhan N., 2018, MNRAS, p. 1627

Henden A. A., Levine S., Terrell D., Welch D. L., 2015, in American Astronomical Society, AAS Meeting 225, id.336.16.

Herwig F., Blöcker T., Langer N., Driebe T., 1999, A\&A, 349, L5

Hollands M. A., Koester D., Alekseev V., Herbert E. L., Gänsicke B. T., 2017, MNRAS, 467, 4970

Jones E., Oliphant T., Peterson P., et al., 2001, SciPy: Open 
source scientific tools for Python, http://www.scipy.org/

Jura M., 2003, ApJ, 584, L91

Jura M., 2008, AJ, 135, 1785

Jura M., Xu S., 2010, AJ, 140, 1129

Jura M., Xu S., 2012, AJ, 143, 6

Jura M., Young E. D., 2014, AREPS, 42, 45

Jura M., Muno M. P., Farihi J., Zuckerman B., 2009, ApJ, 699, 1473

Kallemeyn G. W., Wasson J. T., 1981, Geochimica Cosmochimica Acta, 45, 1217

Kausch W., et al., 2015, A\&A, 576, A78

Kilic M., Redfield S., 2007, ApJ, 660, 641

Klein B., Jura M., Koester D., Zuckerman B., Melis C., 2010, ApJ, 709, 950

Koester D., 1976, A\&A, 52, 415

Koester D., 2009, A\&A, 498, 517

Koester D., 2010, Mem. Soc. Astron. Italiana, 81, 921

Koester D., Kepler S. O., 2015, A\&A, 583, A86

Koester D., Wilken D., 2006, A\&A, 453, 1051

Koester D., Weidemann V., Zeidler-K.T. E. M., Vauclair G., 1985, A\&A, 142, L5

Koester D., Napiwotzki R., Voss B., Homeier D., Reimers D., 2005, A\&A, 439, 317

Kramida A., Yu. Ralchenko Reader J., and NIST ASD Team 2018, NIST Atomic Spectra Database (ver. 5.5.6), [Online]. Available: https://physics.nist.gov/asd [2018, April 23]. National Institute of Standards and Technology, Gaithersburg, MD.

Lallement R., Vergely J. L., Valette B., Puspitarini L., Eyer L., Casagrande L., 2014, A\&A, 561, A91

Lallement R., et al., 2018, A\&A, 616, A132

Lodders K., 2003, ApJ, 591, 1220

Lodders K., 2010, Astrophysics and Space Science Proceedings, 16,379

MacDonald J., Vennes S., 1991, ApJ, 371, 719

Maíz Apellániz J., Weiler M., 2018, A\&A, 619, A180

Malamud U., Perets H. B., 2016, ApJ, 832, 160

Malamud U., Perets H. B., 2017a, ApJ, 842, 67

Malamud U., Perets H. B., 2017b, ApJ, 849, 8

Manser C. J., et al., 2019, Science, 364, 66

Mauche C. W., Raymond J. C., Cordova F. A., 1988, ApJ, 335, 829

Melis C., Farihi J., Dufour P., Zuckerman B., Burgasser A. J., Bergeron P., Bochanski J., Simcoe R., 2011, ApJ, 732, 90

Melis C., et al., 2012, ApJ, 751, L4

Metzger B. D., Rafikov R. R., Bochkarev K. V., 2012, MNRAS, 423,505

Mustill A. J., Villaver E., Veras D., Gänsicke B. T., Bonsor A., 2018, MNRAS, 476, 3939

Nave G., et al., 2019, in BAAS. p. 1

Nelan E. P., Wegner G., 1985, ApJ, 289, L31

O’Donoghue D., Kilkenny D., Koen C., Hambly N., MacGillivray H., Stobie R. S., 2013, MNRAS, 431, 240

Raddi R., Gänsicke B. T., Koester D., Farihi J., Hermes J. J., Scaringi S., Breedt E., Girven J., 2015, MNRAS, 450, 2083

Raddi R., et al., 2017, MNRAS, 472, 4173

Rocchetto M., Farihi J., Gänsicke B. T., Bergfors C., 2015, MNRAS, 449, 574

Rolland B., Bergeron P., Fontaine G., 2018, ApJ, 857, 56

Rolland B., Bergeron P., Fontaine G., 2020, arXiv e-prints, p. arXiv:2001.01085

Skrutskie M. F., et al., 2006, AJ, 131, 1163

Smette A., et al., 2015, A\&A, 576, A77

Stephan A. P., Naoz S., Zuckerman B., 2017, ApJ, 844, L16

Sutherland W., et al., 2015, A\&A, 575, A25

Swan A., Farihi J., Wilson T. G., 2019a, MNRAS, 484, L109

Swan A., Farihi J., Koester D., Hollands M., Parsons S., Cauley

P. W., Redfield S., Gänsicke B. T., 2019b, MNRAS, 490, 202
Tremblay P.-E., Bergeron P., 2008, ApJ, 672, 1144

Veras D., Leinhardt Z. M., Bonsor A., Gänsicke B. T., 2014a, MNRAS, 445, 2244

Veras D., Shannon A., Gänsicke B. T., 2014b, MNRAS, 445, 4175

Veras D., Leinhardt Z. M., Eggl S., Gänsicke B. T., 2015, MNRAS, 451, 3453

Vernet J., et al., 2011, A\&A, 536, A105

Voss B., Koester D., Napiwotzki R., Christlieb N., Reimers D., 2007, A\&A, 470, 1079

Wall R. E., Kilic M., Bergeron P., Rolland B., Genest-Beaulieu C., Gianninas A., 2019, MNRAS, 489, 5046

Wang T.-g., et al., 2019, ApJ, 886, L5

Wasson J. T., Kallemeyn G. W., 1988, Philosophical Transactions of the Royal Society of London Series A, 325, 535

Wegner G., 1982, ApJ, 261, L87

Wegner G., 1984, AJ, 89, 1050

Werner M. W., et al., 2004, ApJS, 154, 1

Wilson D. J., Gänsicke B. T., Koester D., Raddi R., Breedt E., Southworth J., Parsons S. G., 2014, MNRAS, 445, 1878

Wilson D. J., Gänsicke B. T., Farihi J., Koester D., 2016, MNRAS, 459, 3282

Wilson T. G., Farihi J., Gänsicke B. T., Swan A., 2019, MNRAS, 487,133

Xu S., Jura M., 2014, ApJ, 792, L39

Xu S., Jura M., Klein B., Koester D., Zuckerman B., 2013, ApJ, 766,132

Xu S., Zuckerman B., Dufour P., Young E. D., Klein B., Jura M., 2017, ApJ, 836, L7

Xu S., et al., 2018, ApJ, 866, 108

Yeomans D. K., et al., 1997, Science, 278, 2106

Zuckerman B., Koester D., Melis C., Hansen B. M., Jura M., 2007, ApJ, 671, 872

von Hippel T., Kuchner M. J., Kilic M., Mullally F., Reach W. T., 2007, ApJ, 662, 544

This paper has been typeset from a $\mathrm{T}_{\mathrm{EX}} / \mathrm{IAT}_{\mathrm{EX}}$ file prepared by the author. 